\title{
Self-Organizing Multi-layer Fuzzy Polynomial Neural Networks Based on Genetic Optimization
}

\author{
Sung-Kwun $\mathrm{Oh}^{1}$, Witold Pedrycz ${ }^{2}$, Hyun-Ki Kim ${ }^{3}$, and Jong-Beom Lee ${ }^{1}$ \\ ${ }^{1}$ Department of Electrical Electronic and Information Engineering, Wonkwang University, \\ 344-2, Shinyong-Dong, Iksan, Chon-Buk, 570-749, South Korea \\ \{ohsk, ipower\} @wonkwang.ac.kr \\ http: / / autosys.wonkwang.ac.kr \\ ${ }^{2}$ Department of Electrical and Computer Engineering, University of Alberta, Edmonton, \\ AB T6G 2G6, Canada \\ and Systems Research Institute, Polish Academy of Sciences, Warsaw, Poland \\ pedrycz@ee.ualberta.ca \\ ${ }^{3}$ Department of Electrical Engineering, University of Suwon, South Korea \\ hkkimasuwon.ac.kr
}

\begin{abstract}
In this paper, we introduce a new topology of Fuzzy Polynomial Neural Networks (FPNN) that is based on a genetically optimized multilayer perceptron with fuzzy polynomial neurons (FPNs) and discuss its comprehensive design methodology involving mechanisms of genetic optimization, especially genetic algorithms (GAs). The proposed FPNN gives rise to a structurally optimized structure and comes with a substantial level of flexibility in comparison to the one we encounter in conventional FPNNs. The structural optimization is realized via GAs whereas in case of the parametric optimization we proceed with a standard least square method-based learning. Through the consecutive process of such structural and parametric optimization, an optimized and flexible fuzzy neural network is generated in a dynamic fashion. The performance of the proposed gFPNN is quantified through experimentation that exploits standard data already used in fuzzy modeling. These results reveal superiority of the proposed networks over the existing fuzzy and neural models.
\end{abstract}

\section{Introduction}

Recently, a lots of attention has been directed towards advanced techniques of complex system modeling. While neural networks, fuzzy sets and evolutionary computing as the technologies of Computational Intelligence (CI) have expanded and enriched a field of modeling quite immensely, they have also gave rise to a number of new methodological issues and increased our awareness about tradeoffs one has to make in system modeling [1-4]. The most successful approaches to hybridize fuzzy systems with learning and adaptation have been made in the realm of CI. Especially neural fuzzy systems and genetic fuzzy systems hybridize the approximate inference method of fuzzy systems with the learning capabilities of neural networks and evolutionary algorithms [5]. As one of the representative design approaches which are advanced tools, a family of fuzzy polynomial neuron (FPN)-based SOPNN(called "FPNN" as a 
new category of neuro-fuzzy networks)[6] were introduced to build predictive models for such highly nonlinear systems. The FPNN algorithm exhibits some tendency to produce overly complex networks as well as a repetitive computation load by the trial and error method and/or the repetitive parameter adjustment by designer like in case of the original GMDH algorithm. In this study, in addressing the above problems with the conventional SOPNN (especially, FPN-based SOPNN called "FPNN" [6, 9]) as well as the GMDH algorithm, we introduce a new genetic design approach; as a consequence we will be referring to these networks as GA-based FPNN (to be called "gFPNN"). The determination of the optimal values of the parameters available within an individual FPN (viz. the number of input variables, the order of the polynomial, and input variables) leads to a structurally and parametrically optimized network.

\section{The Architecture and Development of Fuzzy Polynomial Neural Networks (FPNN)}

\subsection{FPNN Based on Fuzzy Polynomial Neurons (FPNs)}

The FPN consists of two basic functional modules. The first one, labeled by $\mathbf{F}$, is a collection of fuzzy sets that form an interface between the input numeric variables and the processing part realized by the neuron. The second module (denoted here by P) is about the function - based nonlinear (polynomial) processing. This nonlinear processing involves some input variables. In other words, FPN realizes a family of multiple-input single-output rules. Each rule reads in the form

$$
\text { If } x_{p} \text { is } \boldsymbol{A}_{l} \text { and } x_{q} \text { is } \boldsymbol{B}_{\boldsymbol{k}} \text { then } z \text { is } P_{l k}\left(x_{i}, x_{j}, \boldsymbol{a}_{l k}\right)
$$

where $\boldsymbol{a}_{l k}$ is a vector of the parameters of the conclusion part of the rule while $P_{l k}\left(x_{i}, x_{j}\right.$, $\boldsymbol{a}_{l k}$ ) denotes the regression polynomial forming the consequence part of the fuzzy rule which uses several types of high-order polynomials besides the constant function forming the simplest version of the consequence; refer to Table 1. The activation levels of the rules contribute to the output of the FPN being computed as a weighted average of the individual condition parts (functional transformations) $P_{K}$ (note that the index of the rule, namely " $K$ " is a shorthand notation for the two indexes of fuzzy sets used in the rule (1), that is $K=(1, \mathrm{k})$ ).

$$
\begin{gathered}
z=\sum_{K=1}^{\text {all } \text { rules }} \mu_{K} P_{K}\left(x_{i}, x_{j}, \boldsymbol{a}_{K}\right) / \sum_{K=1}^{\text {all rules }} \mu_{K}=\sum_{K=1}^{\text {all rules }} \tilde{\mu}_{K} P_{K}\left(x_{i}, x_{j}, \boldsymbol{a}_{K}\right) \\
\tilde{\mu}_{K}=\frac{\mu_{K}}{\sum_{L=1}^{\text {all rules }}}
\end{gathered}
$$


Table 1. Different forms of the regression polynomials standing in the consequence part of the fuzzy

\begin{tabular}{|c|c|c|c|}
\hline Order of the polynotint & 1 & 2 & 3 \\
\hline $0($ Type 1$)$ & Constant & Constant & Constant. \\
\hline 1 (1уурс 2) & Lincar & Bilincar & 'Irilinear \\
\hline 2 (Турс 3) & \multirow{2}{*}{ Quadratic } & Biquadratic-1 & 'Iriquadratic-1 \\
\hline 2 (Туре 4) & & Biquadralic-2 & Triquadralic-2 \\
\hline
\end{tabular}

1: Basic type, 2: Moxdified type

\subsection{Genetic Optimization of FPNN}

GAs is a stochastic search technique based on the principles of evolution, natural selection, and genetic recombination by simulating "survival of the fittest" in a population of potential solutions(individuals) to the problem at hand [7]. For the optimization of the FPNN model, GA uses the serial method of binary type, roulette-wheel used in the selection process, one-point crossover in the crossover operation, and a binary inversion (complementation) operation in the mutation operator. To retain the best individual and carry it over to the next generation, we use elitist strategy [8].

\section{The Algorithms and Design Procedure of Genetically Optimized FPNN}

The framework of the design procedure of the Fuzzy Polynomial Neural Networks (FPNN) based on genetically optimized multi-layer perceptron architecture comprises the following steps.

[Step 1] Determine system's input variables

[Step 2] Form training and testing data

The input-output data set $\left(\boldsymbol{x}_{i}, y_{i}\right)=\left(x_{1 i}, x_{2 i}, \ldots, x_{n i}, y_{i}\right), i=1,2, \ldots, N$ is divided into two parts, that is, a training and testing dataset.

[Step 3] Decide initial information for constructing the FPNN structure

[Step 4] Decide FPN structure using genetic design

When it comes to the organization of the chromosome representing (mapping) the structure of the FPNN, we divide the chromosome to be used for genetic optimization into three sub-chromosomes. The $1^{\text {st }}$ sub-chromosome contains the number of input variables, the $2^{\text {nd }}$ sub-chromosome involves the order of the polynomial of the node, and the $3^{\text {rd }}$ sub-chromosome (remaining bits) contains input variables coming to the corresponding node (FPN). All these elements are optimized when running the GA.

[Step 5] Carry out fuzzy inference and coefficient parameters estimation for fuzzy identification in the selected node (FPN)

Regression polynomials (polynomial and in the specific case, a constant value) standing in the conclusion part of fuzzy rules are given as different types of Type 1 , 2,3 , or 4 , see Table 1 . In each fuzzy inference, we consider two types of membership 
functions, namely triangular and Gaussian-like membership functions. The consequence parameters are produced by the standard least squares method

[Step 6] Select nodes (FPNs) with the best predictive capability and construct their corresponding layer

The generation process can be organized as the following sequence of steps

Sub-step 1) We set up initial genetic information necessary for generation of the FPNN architecture.

Sub-step 2) The nodes (FPNs) are generated through the genetic design.

Sub-step 3) We calculate the fitness function. The fitness function reads as

$$
F(\text { fitness function })=1 /(1+E P I)
$$

where EPI denotes the performance index for the testing data (or validation data).

Sub-step 4) To move on to the next generation, we carry out selection, crossover, and mutation operation using genetic initial information and the fitness values obtained via sub-step 3.

Sub-step 5) We choose several FPNs characterized by the best fitness values. Here, we use the pre-defined number $W$ of FPNs with better predictive capability that need to be preserved to assure an optimal operation at the next iteration of the FPNN algorithm. The outputs of the retained nodes (FPNs) serve as inputs to the next layer of the network. There are two cases as to the number of the retained FPNs, that is

(i) If $W^{*}<W$, then the number of the nodes retained for the next layer is equal to $z$. Here, $W^{*}$ denotes the number of the retained nodes in each layer that nodes with the duplicated fitness values are moved.

(ii) If $W^{*} \geq W$, then for the next layer, the number of the retained nodes is equal to $W$.

Sub-step 6) For the elitist strategy, we select the node that has the highest fitness value among the selected nodes $(W)$.

Sub-step 7) We generate new populations of the next generation using operators of GAs obtained from Sub-step 4. We use the elitist strategy. This sub-step carries out by repeating sub-step 2-6.

Sub-step 8) We combine the nodes ( $W$ populations) obtained in the previous generation with the nodes ( $W$ populations) obtained in the current generation.

Sub-step 9) Until the last generation, this sub-step carries out by repeating $\mathbf{s u b}$ step 7-8.

[Step 7] Check the termination criterion

As far as the performance index is concerned (that reflects a numeric accuracy of the layers), a termination is straightforward and comes in the form,

$$
F_{1} \leq F_{*}
$$

Where, $F_{1}$ denotes a maximal fitness value occurring at the current layer whereas $F$, stands for a maximal fitness value that occurred at the previous layer. In this study, we use a measure (performance index) that is the Root Mean Squared Error (RMSE).

$$
E(P I \text { or } E P I)=\sqrt{\frac{1}{N} \sum_{p=1}^{N}\left(y_{p}-\hat{y}_{p}\right)^{2}}
$$


[Step 8] Determine new input variables for the next layer

If (5) has not been met, the model is expanded. The outputs of the preserved nodes $\left(z_{1 i}, z_{2 i}, \ldots, z_{W i}\right)$ serves as new inputs to the next layer $\left(x_{1 j}, x_{2 j}, \ldots, x_{W j}\right)(j=i+1)$. This is captured by the expression

$$
x_{1 j}=z_{1 i}, x_{2 j}=z_{2 i}, \ldots, x_{w j}=z_{w i}
$$

The FPNN algorithm is carried out by repeating steps 4-8.

\section{Experimental Studies}

The performance of the GA-based FPNN is illustrated with the aid of well-known and widely used dataset of the chaotic Mackey-Glass time series [10-17].

The time series is generated by the chaotic Mackey-Glass differential delay equation comes in the form

$$
\dot{x}(t)=\frac{0.2 x(t-\tau)}{1+x^{10}(t-\tau)}-0.1 x(t)
$$

To obtain the time series value at each integer point, we applied the fourth-order Runge-Kutta method to find the numerical solution to (8). From the Mackey-Glass time series $x(t)$, we extracted 1000 input-output data pairs in the following format:

$$
[x(t-18), x(t-12), x(t-6), x(t) ; x(t+6)]
$$

where, $\mathrm{t}=118$ to 1117 . The first 500 pairs were used as the training data set while the remaining 500 pairs formed the testing data set. To come up with a quantitative evaluation of the network, we use the standard RMSE performance index as given by (6). Table 2 summarizes the list of parameters used in the genetic optimization of the network.

Table 2. Summary of the parameters of the genetic optimization

\begin{tabular}{|c||c||c||c|}
\hline \multicolumn{1}{|c||}{} & Parameters & $1^{\text {st }}$ layer & $2^{\text {nd }}$ to $5^{\text {th }}$ layer \\
\hline \hline \multirow{4}{*}{ GA } & Maximum generation & 100 & 100 \\
\cline { 2 - 4 } & Total population size & 60 & 60 \\
\cline { 2 - 4 } & Selected population size $(W)$ & 30 & 30 \\
\cline { 2 - 4 } & Crossover rate & 0.65 & 0.65 \\
\cline { 2 - 4 } & Mutation rate & 0.1 & 0.1 \\
\hline \multirow{4}{*}{ FPNN } & String length & $3+3+30$ & $3+3+30$ \\
\cline { 2 - 4 } & Maximal no.(Max) of inputs to be selected & $1 \leq l \leq \operatorname{Max}(2 \sim 5)$ & $1 \leq l \leq \mathrm{Max}(2 \sim 5)$ \\
\cline { 2 - 4 } & $\begin{array}{c}\text { Polynomial type (Type T) of the consequent } \\
\text { part of fuzzy rules }\end{array}$ & $1 \leq \mathrm{T} \leq 4$ & $1 \leq \mathrm{T} \leq 4$ \\
\cline { 2 - 4 } & Consequent input type to be used for Type T $(*)$ & Type T* & Type T \\
\cline { 2 - 4 } & \multirow{2}{*}{ Membership Function (MF) type } & Triangular & Triangular \\
\cline { 2 - 4 } & No. of MFs per input & Gaussian & Gaussian \\
\hline
\end{tabular}

$l, \mathrm{P}, \mathrm{Max}$ : integers, $\mathrm{T}^{*}$ means that entire system inputs are used for the polynomial in the conclusion part of the rules. 
Table 3 summarizes the performance of the $1^{\text {st }}$ and the $2^{\text {nd }}$ layer of the network when changing the maximal number of inputs to be selected; here Max was set up to 2 through 5 .

Table 3. Performance index of the network of each layer versus the increase of maximal number of inputs to be selected

(a) Triangular MF

\begin{tabular}{|c|c|c|c|c|c|c|c|c|c|}
\hline \multirow[t]{2}{*}{ Max } & \multicolumn{4}{|c|}{$1^{\text {st }}$ layer } & \multicolumn{5}{|c|}{$2^{\text {nd }}$ layer } \\
\hline & Noc & de & $\begin{array}{ll}\mathrm{T} & \mathrm{PI} \\
\end{array}$ & EPI & $\mathrm{Nod}$ & $\mathrm{de}$ & $\mathrm{T} \quad \mathrm{P}$ & PI & \\
\hline 2 & 1 & 2 & \begin{tabular}{l|l|}
30.0056 \\
\end{tabular} & 0.0054 & & 29 & \begin{tabular}{|l|l|}
4 & 0.00 \\
\end{tabular} & $050 \mid 0.1$ & \\
\hline 3 & \begin{tabular}{l|l|}
1 & 2 \\
\end{tabular} & 3 & \begin{tabular}{l|l|}
30.0040 \\
\end{tabular} & 0.0040 & \begin{tabular}{l|l|}
2 & 16 \\
\end{tabular} & 25 & $2 \mid 0.00$ & 03 & \\
\hline 4 & & \begin{tabular}{|l|l|}
3 & 4 \\
\end{tabular} & 0.0017 & \begin{tabular}{|l|}
0.0016 \\
\end{tabular} & \begin{tabular}{|l|l|l}
9 & 12 & 2 \\
\end{tabular} & \begin{tabular}{|l|l|}
22 & 28 \\
\end{tabular} & \begin{tabular}{|l|l}
3 & 0.00 \\
\end{tabular} & \begin{tabular}{l|l}
015 & 0.1 \\
\end{tabular} & \\
\hline & & & 000 & & & & 200 & & \\
\hline
\end{tabular}

(b) Gaussian-like MF

\begin{tabular}{|c|c|c|c|c|c|c|c|}
\hline \multicolumn{5}{|c|}{$1^{\text {st }}$ layer } & \multicolumn{3}{|c|}{$2^{\text {nd }}$ layer } \\
\hline \multicolumn{3}{|c|}{ Node } & \begin{tabular}{|l|l|}
$T$ & $P I$ \\
\end{tabular} & EPI & $\mathrm{Noc}$ & $\mathrm{de}$ & \begin{tabular}{l|l|l|}
$\mathrm{T}$ & $\mathrm{PI}$ & $\mathrm{EP}$ \\
\end{tabular} \\
\hline 1 & & 2 & \begin{tabular}{|l|l|}
3 & 0.0028 \\
\end{tabular} & \begin{tabular}{|l|l|l|}
0.0027 \\
\end{tabular} & 19 & 26 & \begin{tabular}{l|l|l|}
3 & 0.00260 .002
\end{tabular} \\
\hline & 2 & 4 & \begin{tabular}{l|l|}
3 & 0.0014 \\
\end{tabular} & 0.0013 & \begin{tabular}{l|l}
15 & 25 \\
\end{tabular} & 27 & \begin{tabular}{|l|l|l|l}
4 & 0.0012 & 0.00
\end{tabular} \\
\hline 1 & \begin{tabular}{|l|l|}
2 & 3 \\
\end{tabular} & 4 & \begin{tabular}{|l|l|}
3 & 0.0005 \\
\end{tabular} & 0.0006 & \begin{tabular}{|l|l|l|}
10 & 23 & 2 \\
\end{tabular} & 2429 & \begin{tabular}{|l|l|l|}
2 & 0.0004 & 0.00
\end{tabular} \\
\hline & 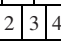 & $\begin{array}{lll}4 & 0\end{array}$ & & & & & $4[0.000$ \\
\hline
\end{tabular}

Fig. 1 depicts the performance index of each layer of gFPNN according to the increase of maximal number of inputs to be selected. Fig. 2(a) illustrates the detailed optimal topologies of gFPNN for 1 layer when using Max=4 and triangular MF. And also Fig. 2(b) illustrates the detailed optimal topology of gFPNN for 1 layer in case of using Max= 3 and Gaussian-like MF.
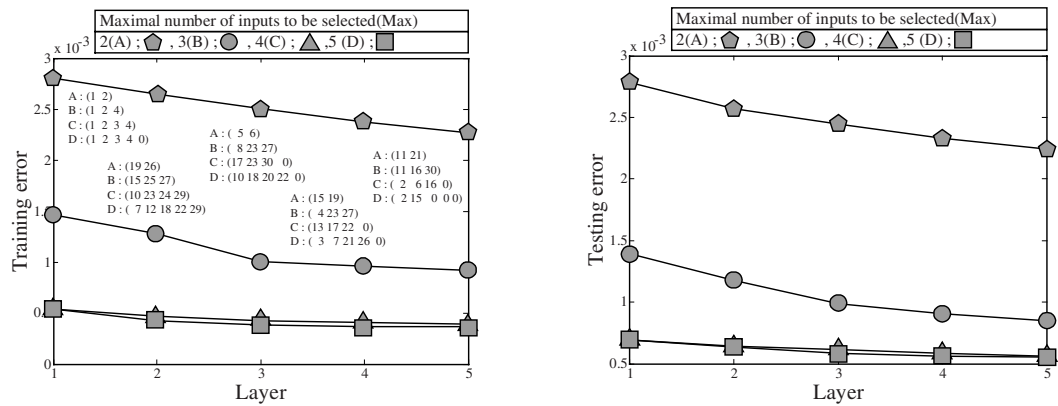

Fig. 1. Performance index of gFPNN with respect to the increase of number of layers (Gaussian-like MF)

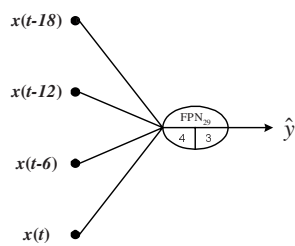

(a) Triangular MF (1 layer)

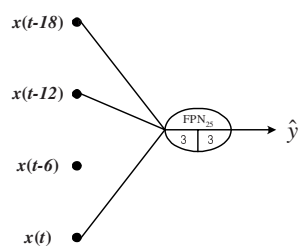

(b) Gaussian-like MF

Fig. 2. Genetically optimized FPNN(gFPNN) architecture

Table 4 gives a comparative summary of the network with other models. 
Table 4. Comparative analysis of the performance of the network; considered are models reported in the literature

\begin{tabular}{|c|c|c|c|c|c|c|}
\hline & \multirow{2}{*}{\multicolumn{2}{|c|}{ Model }} & \multicolumn{4}{|c|}{ Performance index } \\
\hline & & & PI & $\mathrm{PI}$ & EPI & $\mathrm{NDEI}^{*}$ \\
\hline & & & 0.044 & & & \\
\hline & Wang's mod & & 0.013 & & & \\
\hline & & & 0.010 & & & \\
\hline & ded-correlati & $\mathrm{NN}[11]$ & & & & 0.06 \\
\hline & kpropagation & $\mathrm{P}[11]$ & & & & 0.02 \\
\hline & order polyno & al[11] & & & & 0.04 \\
\hline & ANFIS[1 & & & 0.0016 & 0.0015 & 0.007 \\
\hline & FNN model & & & 0.014 & 0.009 & \\
\hline & rent neural $n$ & ork[14] & 0.0138 & & & \\
\hline & Basic & Case 1 & & 0.0011 & 0.0011 & 0.005 \\
\hline SONA**[15] & ( $5^{\text {th }}$ layer $)$ & Case 2 & & 0.0027 & 0.0028 & 0.011 \\
\hline SONN & Modified & Case 1 & & 0.0012 & 0.0011 & 0.005 \\
\hline & ( $5^{\text {th }}$ layer $)$ & Case 2 & & 0.0038 & 0.0038 & 0.016 \\
\hline & & $2^{\text {nd }}$ layer $(\operatorname{Max}=4)$ & 0.0014 & 0.0015 & 0.0014 & 0.0061 \\
\hline & & $5^{\text {th }}$ layer(Max=4) & 0.0010 & 0.0011 & 0.0011 & 0.0045 \\
\hline & Triangular & $2^{\text {nd }}$ layer $(\operatorname{Max}=5)$ & 0.0011 & 0.0014 & 0.0013 & 0.0051 \\
\hline $\begin{array}{c}\text { Proposed } \\
\text { gFPNN }\end{array}$ & & $5^{\text {th }}$ layer(Max=5) & 0.0007 & 0.0009 & 0.0010 & 0.0031 \\
\hline & & $1^{\text {st }}$ layer(Max=3) & 0.0014 & 0.0014 & 0.0013 & 0.0064 \\
\hline & Gaussian & $1^{\text {st }}$ layer(Max=4) & 0.0005 & 0.0005 & 0.0006 & 0.0023 \\
\hline & & $1^{\text {st }}$ layer(Max=5) & 0.0005 & 0.0005 & 0.0006 & 0.0023 \\
\hline
\end{tabular}

"Non-dimensional error index (NDEI) as used in [16] is defined as the root mean square errors divided by the standard deviation of the target series. ** is called "conventional optimized FPNN".

\section{Concluding Remarks}

In this study, the GA-based design procedure of Fuzzy Polynomial Neural Networks (FPNN) along with their architectural considerations has been investigated. The design methodology comes as a hybrid structural optimization and parametric learning being viewed as two fundamental phases of the design process. The GMDH method is now comprised of both a structural phase such as a self-organizing and an evolutionary algorithm (rooted in natural law of survival of the fittest), and the ensuing parametric phase of the Least Square Estimation (LSE)-based learning. The comprehensive experimental studies involving well-known datasets quantify a superb performance of the network in comparison to the existing fuzzy and neuro-fuzzy models. Most importantly, through the proposed framework of genetic optimization we can efficiently search for the optimal network architecture (structurally and parametrically optimized network) and this becomes crucial in improving the performance of the resulting model. 
Acknowledgements. This work has been supported by EESRI(R-2003-B-274), which is funded by MOCIE (Ministry of Commerce, Industry and Energy).

\section{References}

1. V. Cherkassky, D. Gehring, F. Mulier.: Comparison of adaptive methods for function estimation from samples. IEEE Trans. Neural Networks, 7 (1996) 969-984

2. J. A. Dickerson, B. Kosko.: Fuzzy function approximation with ellipsoidal rules. IEEE Trans. Syst., Man, Cybernetics. Part B. 26 (1996) 542-560

3. V. Sommer, P. Tobias, D. Kohl, H. Sundgren, L. Lundstrom.: Neural networks and abductive networks for chemical sensor signals: A case comparison. Sensors and Actuators B. 28 (1995) 217-222

4. S. Kleinsteuber, N. Sepehri.: A polynomial network modeling approach to a class of large-scale hydraulic systems. Computers Elect. Eng. 22 (1996) 151-168

5. O. Cordon, et al.: Ten years of genetic fuzzy systems: current framework and new trends. Fuzzy Sets and Systems. 2003(in press)

6. S.-K. Oh, W. Pedrycz.: Self-organizing Polynomial Neural Networks Based on Polynomial and Fuzzy Polynomial Neurons: Analysis and Design. Fuzzy Sets and Systems. 142(2) (2003) 163-198

7. Michalewicz, Z.: Genetic Algorithms + Data Structures = Evolution Programs. 3rd edn Springer-Verlag, Berlin Heidelberg New York (1996)

8. D. Jong, K. A.: Are Genetic Algorithms Function Optimizers?. Parallel Problem Solving from Nature 2, Manner, R. and Manderick, B. eds., North-Holland, Amsterdam

9. S.-K. Oh, W. Pedrycz.: Fuzzy Polynomial Neuron-Based Self-Organizing Neural Networks. Int. J. of General Systems, 32(2003) 237-250

10. L. X. Wang, J. M. Mendel.: Generating fuzzy rules from numerical data with applications. IEEE Trans. Systems, Man, Cybern. 22 (1992) 1414-1427

11. R. S. Crowder III.: Predicting the Mackey-Glass time series with cascade-correlation learning. In: D. Touretzky, G. Hinton, and T. Sejnowski, editors, Proceedings of the 1990 Connectionist Models Summer School, Carnegic Mellon University, (1990) 117-123

12. J. S. R. Jang.: ANFIS: Adaptive-Network-Based Fuzzy Inference System. IEEE Trans. System, Man, and Cybern. 23 (1993) 665-685

13. L. P. Maguire, B. Roche, T. M. McGinnity, L. J. McDaid.: Predicting a chaotic time series using a fuzzy neural network. Information Sciences. 112(1998) 125-136

14. C. James Li, T. -Y. Huang.: Automatic structure and parameter training methods for modeling of mechanical systems by recurrent neural networks. Applied Mathematical Modeling. 23 (1999) 933-944

15. S.-K. Oh, W. Pedrycz, T.-C. Ahn.: Self-organizing neural networks with fuzzy polynomial neurons. Applied Soft Computing. 2 (2002) 1-10

16. A. S. Lapedes, R. Farber.: Non-linear Signal Processing Using Neural Networks: Prediction and System Modeling. Technical Report LA-UR-87-2662, Los Alamos National Laboratory, Los Alamos, New Mexico 87545, (1987)

17. M. C. Mackey, L. Glass.: Oscillation and chaos in physiological control systems. Science, 197 (1977) 287-289

18. S.-K. Oh, W. Pedrycz.: The design of self-organizing Polynomial Neural Networks. Information Science. 141 (2002) 237-258 
19. S.-K. Oh, W. Pedrycz, B.-J. Park.: Polynomial Neural Networks Architecture: Analysis and Design. Computers and Electrical Engineering. 29 (2003) 703-725

20. B.-J. Park, D.-Y. Lee, S.-K. Oh: Rule-Based Fuzzy Polynomial Neural Networks in Modeling Software Process Data. International Journal of Control, Automation and Systems, 1(3) (2003) 321-331

21. H.-S. Park, S.-K. Oh: Rule-based Fuzzy-Neural Networks Using the Identification Algorithm of GA hybrid Scheme. International Journal of Control, Automation and Systems, 1(1) (2003) 101-110 